\title{
OPEN Quantity of IgG response to SARS-CoV-2 spike glycoprotein predicts pulmonary recovery from COVID-19
}

\author{
Manfred Nairz ${ }^{1 凶}$, Sabina Sahanic ${ }^{1}$, Alex Pizzini ${ }^{1}$, Anna Böhm ${ }^{1}$, Piotr Tymoszuk ${ }^{1}$, \\ Anna-Maria Mitterstiller ${ }^{1}$, Laura von Raffay ${ }^{1}$, Philipp Grubwieser ${ }^{1}$, Rosa Bellmann-Weiler ${ }^{1}$, \\ Sabine Koppelstätter ${ }^{1}$, Andrea Schroll ${ }^{1}$, David Haschka ${ }^{1}$, Martina Zimmermann ${ }^{1}$, \\ Silvia Blunder ${ }^{1}$, Kristina Trattnig ${ }^{1}$, Helene Naschberger ${ }^{1}$, Werner Klotz ${ }^{10}{ }^{1}$, Igor Theurl ${ }^{1}$, \\ Verena Petzer ${ }^{2}$, Clemens Gehrer ${ }^{1}$, John E. Mindur ${ }^{3}$, Anna Luger ${ }^{4}$, Christoph Schwabl ${ }^{4}$, \\ Gerlig Widmann ${ }^{4}$, Günter Weiss ${ }^{1,5}{ }^{1}$, Judith Löffler-Ragg ${ }^{1}$, Ivan Tancevski ${ }^{1}$ \& \\ Thomas Sonnweber ${ }^{1 \bowtie}$
}

The CovILD study is a prospective, multicenter, observational cohort study to systematically follow up patients after coronavirus disease-2019 (COVID-19). We extensively evaluated 145 COVID-19 patients at 3 follow-up visits scheduled for 60, 100, and 180 days after initial confirmed diagnosis based on typical symptoms and a positive reverse transcription-polymerase chain reaction (RT-PCR) for severe acute respiratory syndrome coronavirus-2 (SARS-CoV-2). We employed comprehensive pulmonary function and laboratory tests, including serum concentrations of IgG against the viral spike (S) glycoprotein, and compared the results to clinical data and chest computed tomography (CT). We found that at the 60 day follow-up, 131 of 145 (90.3\%) participants displayed S-specific serum IgG levels above the cut-off threshold. Notably, the highly elevated IgG levels against $S$ glycoprotein positively correlated with biomarkers of immune activation and negatively correlated with pulmonary function and the extent of pulmonary $\mathrm{CT}$ abnormalities. Based on the association between serum $\mathrm{S}$ glycoprotein-specific IgG and clinical outcome, we generated an S-specific Ig G-based recovery score that, when applied in the early convalescent phase, accurately predicted delayed pulmonary recovery after COVID-19. Therefore, we propose that S-specific IgG levels serve as a useful immunological surrogate marker for identifying at-risk individuals with persistent pulmonary injury who may require intensive follow-up care after COVID-19.

Severe acute respiratory syndrome coronavirus 2 (SARS-CoV-2) causes a spectrum of subclinical and clinical manifestations ranging from asymptomatic infection to fatal respiratory and systemic disease $\mathrm{e}^{1-3}$. To date, a plethora of clinical studies were initiated to elucidate the underlying pathophysiology of coronavirus disease 2019 (COVID-19). However, we still lack a comprehensive understanding of the immunopathology of COVID19 and of the effects the immune system exerts on recovery from acute disease.

Host-pathogen interplay occurring in the acute phase of SARS-CoV-2 infection is a major dictating factor in the disease course of COVID-19. This interplay is shaped by local and systemic immune responses against SARS-CoV-2, the spreading of virus in the respiratory tract, and by immune- and pathogen-mediated effects on pulmonary and other systems of the host organism ${ }^{4,5}$. The immune response against respiratory viruses, including SARS-CoV-2, involves rapid innate and subsequent adaptive immune mechanisms ${ }^{6-9}$. To assess the course of COVID-19 in hospitalized patients, innate immune responses can be monitored in the peripheral blood by measuring concentrations of the master inducer of the acute-phase response, interleukin (IL)-6, and

\footnotetext{
${ }^{1}$ Department of Internal Medicine II, Medical University of Innsbruck, Innsbruck, Austria. ${ }^{2}$ Department of Internal Medicine V, Medical University of Innsbruck, Innsbruck, Austria. ${ }^{3}$ Repertoire Immune Medicines, Cambridge, MA, USA. ${ }^{4}$ Department of Radiology, Medical University of Innsbruck, Innsbruck, Austria. ${ }^{5}$ Christian Doppler Laboratory for Iron Metabolism and Anemia Research, Medical University of Innsbruck, Innsbruck, Austria. ${ }^{\square}$ email: manfred.nairz@i-med.ac.at; thomas.sonnweber@i-med.ac.at
} 


\begin{tabular}{|c|c|}
\hline & $\mathrm{N}=145$ \\
\hline \multicolumn{2}{|l|}{ Characteristics } \\
\hline Mean age-year (SD) & $57(14)$ \\
\hline Female sex-no. (\%) & $63(43)$ \\
\hline Mean body mass index $-\mathrm{kg} / \mathrm{m}^{2}(\mathrm{SD})^{\star}$ & $26(5)$ \\
\hline Smoking history—no. (\%) & $57(39)$ \\
\hline \multicolumn{2}{|l|}{ Comorbidities-no. (\%) } \\
\hline None & $33(23)$ \\
\hline Cardiovascular disease & $58(40)$ \\
\hline Hypertension & $44(30)$ \\
\hline Pulmonary disease & $27(19)$ \\
\hline COPD & $8(6)$ \\
\hline Asthma & $10(7)$ \\
\hline Metabolic disease & $63(43)$ \\
\hline Chronic kidney disease & $10(7)$ \\
\hline Chronic liver disease & $8(6)$ \\
\hline Malignancy & $17(12)$ \\
\hline Immunodeficiency $^{*}$ & $10(7)$ \\
\hline Hospitalized-no. (\%) & $109(75)$ \\
\hline \multicolumn{2}{|l|}{ In-hospital treatment ${ }^{\S}$} \\
\hline Oxygen supply-no. (\%) & $72(66)$ \\
\hline Non-invasive ventilation-no. (\%) & $3(3)$ \\
\hline Invasive ventilation - no. $(\%)$ & $29(27)$ \\
\hline
\end{tabular}

Table 1. Demographic and clinical characteristics of COVID-19 patients of the CovILD study. *The bodymass index is the weight kilograms divided by the square of the height in meters. ${ }^{\ddagger}$ Due to disease or ongoing immunosuppressive treatment: renal transplantation $(\mathrm{N}=1)$, psoriasis vulgaris $(\mathrm{N}=1)$, Morbus Hashimoto $(\mathrm{N}=1)$, leukaemia $(\mathrm{N}=1)$, lymphoma $(\mathrm{N}=1)$, gout $(\mathrm{N}=1)$, myasthenia gravis $(\mathrm{N}=1)$, polyarthritis $(\mathrm{N}=3) ;{ }^{\varsigma} \mathrm{All}$ patients needing non-invasive or invasive ventilation were supplied with oxygen before ICU admission, relative numbers depict the treatment of in-hospital patients.

its down-stream effectors, such as C-reactive protein (CRP) and ferritin. Adaptive immune mechanisms, on the other hand, are largely mediated by $\mathrm{B}$ and $\mathrm{T}$ lymphocytes, which give rise to antibody and cytokine-mediated responses $^{10,11}$. Whereas pathogen-specific $\mathrm{T}$ cell responses are laborious to assess in a routine diagnostic setting, $\mathrm{B}$ cell-mediated immunity can be more easily tracked by the quantitative measurement of antibody levels ${ }^{12}$. In this swiftly evolving field, enzyme-linked immunosorbent assays (ELISA) and automated chemiluminescence immunoassays (CLIA) have emerged as the most widely available tests to assess antibody levels. The spike (S) glycoprotein and its receptor-binding domain (RBD) as well as the nucleocapsid (N) protein are common targets of the B cell response and are thus frequently used as antigens to assess humoral immune responses via specific antibody detection ${ }^{13-17}$.

In respiratory infections, three antibody isotypes, $\operatorname{IgM}$, IgG, and $\operatorname{IgA}$, are primarily produced to mediate prompt, long-lasting, and mucosal immunity, respectively ${ }^{18-20}$. In response to SARS-CoV-2 infection, these 3 isotypes emerge early and nearly simultaneously in the serum ${ }^{21}$. Secretory IgA confers strong protection against the virus as assessed by neutralization studies ${ }^{22,23}$, whereas IgG persists in the serum for months, suggesting relatively long-lasting protection ${ }^{24-30}$. Failure to generate sufficient IgG antibodies is linked to reduced survival ${ }^{31}$. Other studies, however, found a positive ${ }^{17,32-34}$ or unclear association ${ }^{35}$ between serum concentrations of IgG antibodies against SARS-CoV-2 and COVID-19 disease severity. These findings are further complicated by the fact that some individuals carry pre-existing antibodies that can neutralize SARS-CoV- ${ }^{36}$, and antibody responses are age-dependent ${ }^{37}$. Hence, the effects of SARS-CoV-2-specific IgG concentrations on the clinical course of COVID-19 remain controversial.

The work presented herein aimed to investigate yet another aspect of COVID-19; more specifically, we assessed whether the quantity of IgG response is associated with lung damage and able to predict pulmonary recovery from symptomatic SARS-CoV-2 infection.

\section{Results}

Clinical and demographic patient characteristics. A total of 145 symptomatic adults who previously suffered from mild to critical COVID-19 were included in the study and attended 3 follow-up appointments scheduled for 60,100 , and 180 days after the initial confirmed diagnosis of COVID-19. The mean age of study participants was 57 years ( $\mathrm{SD} \pm 14$ years) and the majority were male $(57 \%)$. Clinical and demographic characteristics of the CovILD cohort are detailed in Table 1.

Disease severity ranged from mild to critical according to medical treatment need. Specifically, 36 patients (25\%) developed mild disease (outpatient treatment), 37 patients (26\%) developed moderate disease (inpatient 
A

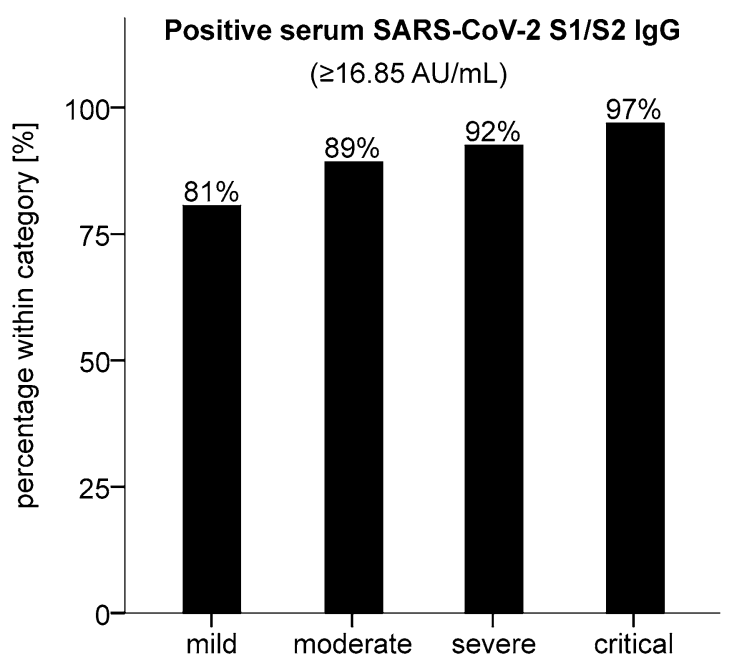

Acute COVID-19 disease severity
B

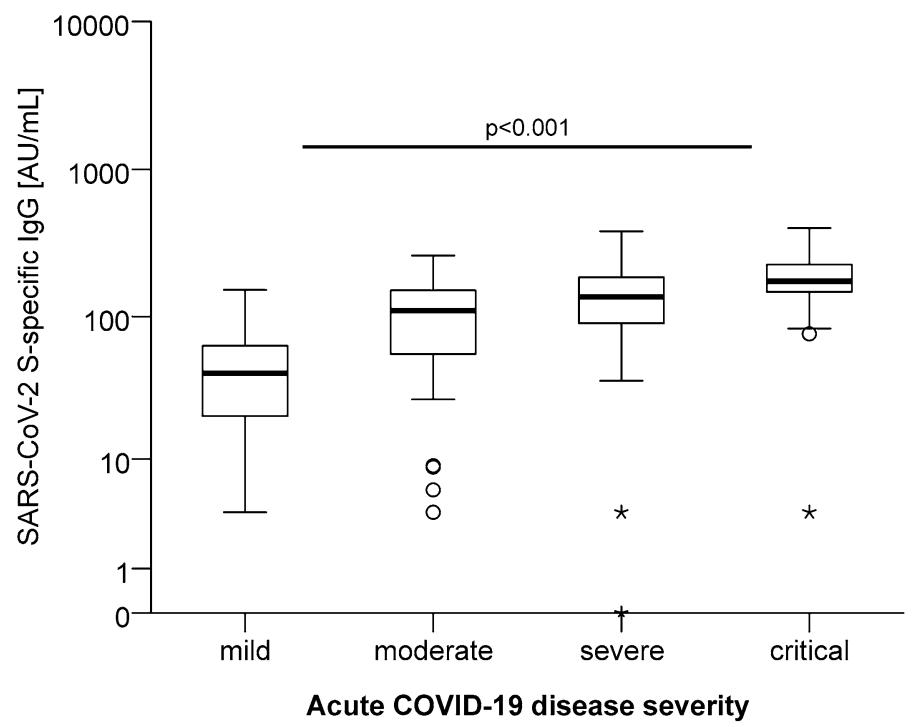

Figure 1. Qualitative and quantitative results for S-specific IgG correlate with the clinical severity of COVID19. Patients were categorized according to clinical severity of acute COVID-19 $\left(\mathrm{N}_{\text {mild }}=36, \mathrm{~N}_{\text {moderate }}=37\right.$, $\mathrm{N}_{\text {severe }}=40, \mathrm{~N}_{\text {critical }}=32$ ). For each clinical category of disease severity, the relative abundance of patients $(\mathbf{A})$ who mounted a substantial IgG response against the $S$ glycoprotein above the cut-off threshold is depicted. The SARS-CoV-2 IgG concentrations were quantified (B) at the 60 days follow-up according to acute disease severity categories. p-values were calculated with the Kruskal-Wallis test.

treatment without respiratory support), 40 patients (28\%) developed severe disease (inpatient treatment with respiratory support), and 32 patients (22\%) developed critical disease and required mechanical ventilation in the intensive care unit (ICU).

S-specific IgG levels correlate with the severity of acute COVID-19. First, we asked whether serum concentrations of IgG specific for the S glycoprotein of SARS-CoV-2 are associated with the disease course. To this end, we used a certified in-vitro diagnostic (IVD) chemiluminescence immunoassay (CLIA) and a validated cut-off threshold of $16.85 \mathrm{AU} / \mathrm{mL}$ to classify study participants, all of whom had been symptomatic and diagnosed prior by reverse transcription-polymerase chain reaction (RT-PCR) as negative or positive for S-specific IgG. According to this qualitative classification, we found that $81 \%$ of patients with mild COVID-19 $(\mathrm{N}=29)$, $89 \%$ with moderate disease $(\mathrm{N}=33), 92 \%$ with severe disease $(\mathrm{N}=37)$, and $97 \%$ with critical disease $(\mathrm{N}=31)$ produced substantial amounts of S-specific IgG (Fig. 1A). Notably, the only patient with critical COVID-19 who did not mount a detectable antibody response against $S$ glycoprotein had received rituximab, a therapeutic CD20-specific monoclonal antibody, eight weeks before SARS-CoV-2 infection. In contrast, none of the other study participants had undergone any treatment known to directly affect antibody production or half-life (e.g. B cell depletion or plasmapheresis).

Second, we compared the quantity of S-specific IgG measured at the 60-day follow-up to the classified severity of acute COVID-19 disease from which the study participants were recovering. We observed that levels of S-specific IgG and severity of COVID-19 were positively correlated (Fig. 1B). Therefore, outpatients with mild disease displayed the lowest antibody levels and ICU patients with critical disease displayed the highest antibody levels at the 60-day follow-up.

Third, we analyzed whether the correlation between S-specific IgG levels and clinical disease course is linked to the exclusive requirement for $\mathrm{O}_{2}$ therapy or intensive care, respectively, and if the differences in quantity of S-specific IgG at the 60 -day follow-up were still present at the 100 - and 180 - day reevaluation. We found that patients requiring $\mathrm{O}_{2}$ therapy had significantly higher S-specific IgG levels at all time-points throughout the observation period in comparison to patients who never required supplemental $\mathrm{O}_{2}$ (Fig. 2A). Similarly, patients admitted to the ICU for critical acute COVID-19 disease displayed significantly higher S-specific IgG levels after 60,100 , and 180 days compared to patients who did not require intensive care (Fig. 2B). Taken together, these data indicate the $S$ glycoprotein-specific IgG response serves as a reliable clinical correlate for acute COVID-19 disease severity, tracking with the degree of patient supportive care.

Correlations of S-specific IgG levels with other biomarkers of COVID-19. Next, we extended our analyses to evaluate known biomarkers associated with COVID-19 disease severity. We saw that at all follow-up visits, patients with persistently elevated IL-6 levels (cut-off: $7 \mathrm{ng} / \mathrm{L}$ ), as well as serum ferritin (cut-off: $400 \mu \mathrm{g} / \mathrm{L}$ ) and hepcidin-25 (cut-off: $20 \mu \mathrm{g} / \mathrm{L}$ ), showed higher S-specific IgG concentrations (Fig. 3). In line with known biomarkers of acute COVID-19 disease severity, these results further highlight the association between the $S$ glycoprotein-specific IgG response and COVID-19 severity. 
A

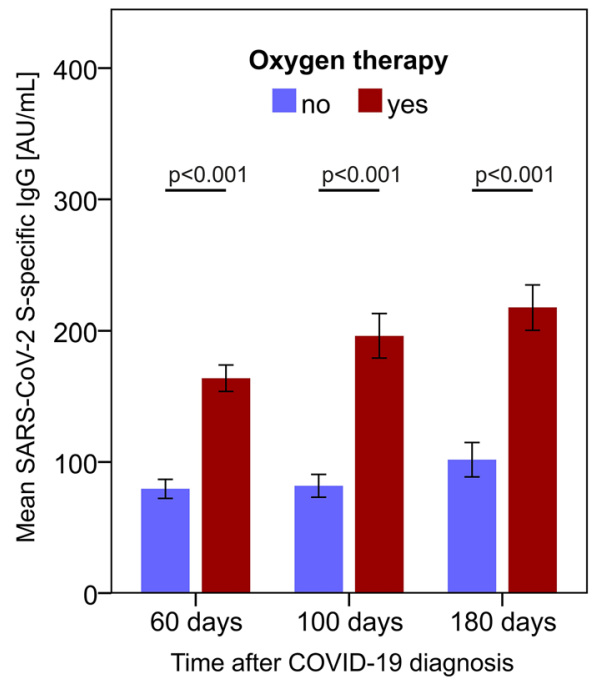

B

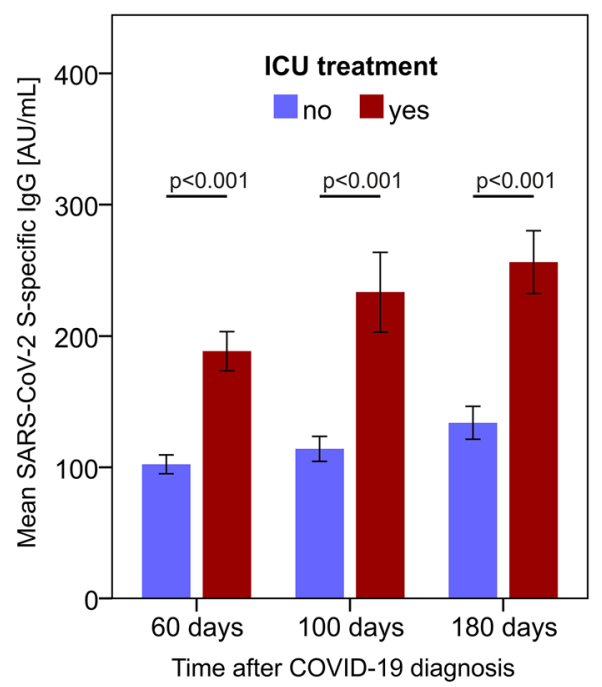

Figure 2. S-specific IgG levels correlate with supplemental $\mathrm{O}_{2}$ requirement and intensive care during acute COVID-19. S-specific IgG serum concentrations are reported according to need for oxygen supply $\left(\mathrm{N}_{\mathrm{Y} / \mathrm{N}}=72 / 73\right)$ or ICU treatment $\left(\mathrm{N}_{\mathrm{Y} / \mathrm{N}}=32 / 113\right)$ during acute COVID-19. P-values were calculated with the Mann-Whitney- $U$ test. $\mathrm{N}_{60 \text { days }}=145 ; \mathrm{N}_{100 \text { days }}=135 ; \mathrm{N}_{180 \text { days }}=118$.

A

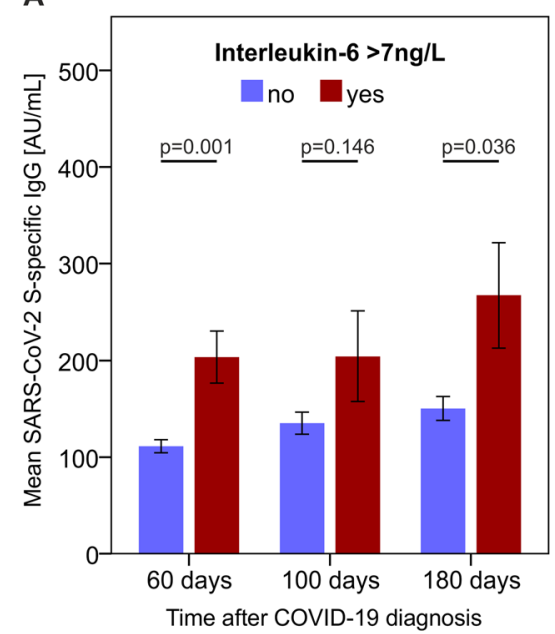

B

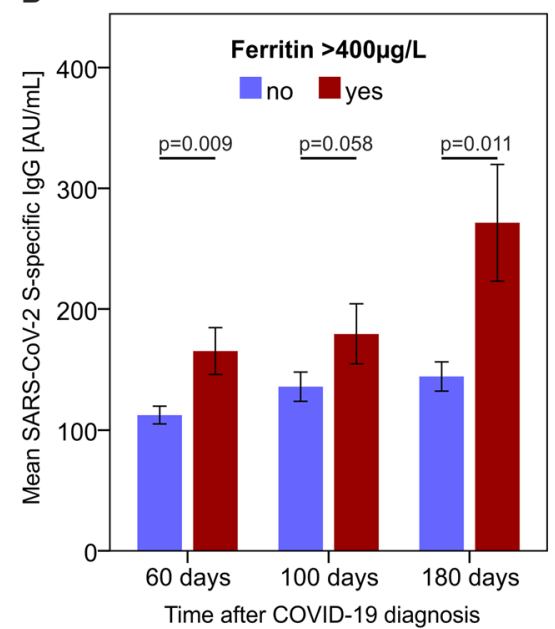

C

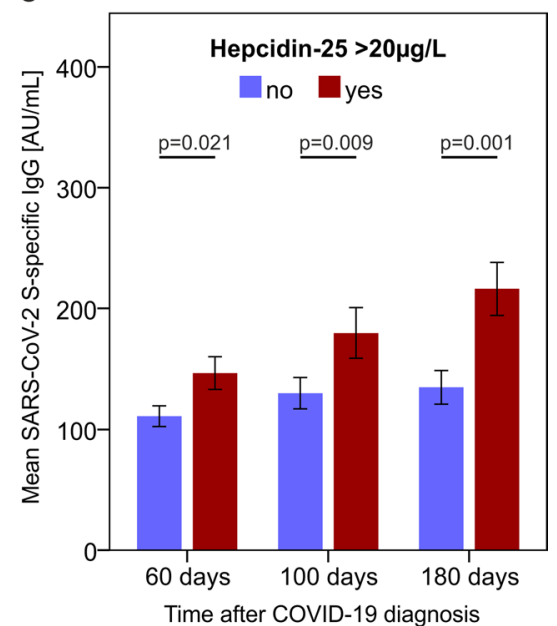

Figure 3. S-specific IgG is associated with elevated serum IL-6 levels, ferritin, and hepcidin-25. Patients with persistingly elevated IL-6 (cut-off: $7 \mathrm{ng} / \mathrm{L})(\mathbf{A})$, ferritin $(>400 \mu \mathrm{g} / \mathrm{L})(\mathbf{B})$, and hepcidin-25 (> $20 \mu \mathrm{g} / \mathrm{L})(\mathbf{C})$ demonstrate significantly higher S-specific IgG concentrations compared to individuals without elevated IL-6 at follow-up. $12 \%, 6 \%$, and $4 \%$ of patients demonstrated increased IL-6 levels at the 60-day, 100-day, and 180-day follow-up, respectively. $\mathrm{N}_{60 \text { days }}=145 ; \mathrm{N}_{100 \text { days }}=135 ; \mathrm{N}_{180 \text { days }}=118$.

Levels of S-specific IgG are associated with persistently impaired pulmonary function. Given the relation between serum S-specific IgG concentrations and COVID-19 severity, we then asked if S-specific SARS-CoV-2 IgG also correlated with pulmonary recovery. Thus, we searched for a correlation between concentrations of S-specific IgG and pulmonary function based on functional lung assessments performed by body plethysmography. Notably, we found that patients with impaired lung function, including measurements of forced expiratory volume in $1 \mathrm{~s}$ (FEV1; cut-off $80 \%$ of the calculated normal value), forced vital capacity (FVC; cut-off $80 \%$ of normal), FEV1/FVC (cut-off $70 \%$ ) or total lung capacity (TLC; cut-off $80 \%$ of normal), as well as a reduction of the diffusion capacity for carbon monoxide (DLCO) below normal levels (cut-off $80 \%$ of the normal level calculated from sex, age, and height) and hypoxia (arterial $\mathrm{pO}_{2}$; cut-off $65 \mathrm{mmHg}$ ), were related to increased S-specific IgG levels at the 60-day follow-up (Fig. 4).

Levels of S-specific IgG are associated with persistent abnormalities in pulmonary computed tomography. Next, we looked for possible correlations between S-specific IgG levels and structural pulmo- 


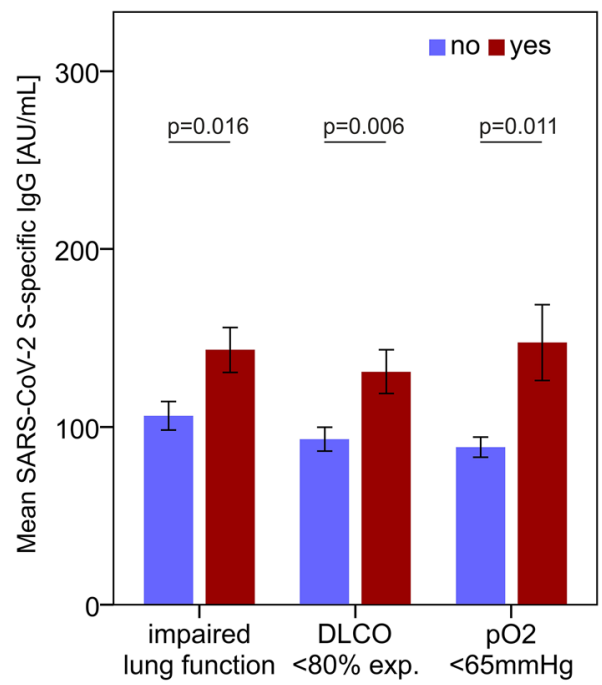

Figure 4. S-specific IgG is related to pulmonary function and hypoxia. S-specific IgG levels are increased in patients with impaired lung function and hypoxia at the 60-day follow-up. Serum S-specific IgG concentrations in patients with any impairment of lung function (including reduced FEV1, FVC, TLC, or DLCO), reduction of diffusion capacity for carbon monoxide (DLCO), or significant hypoxia (defined by an arterial $\mathrm{pO}_{2}<65 \mathrm{mmHg}$ ) are shown. P-values were calculated with the Mann-Whitney $\mathrm{U}$ test. $\mathrm{N}_{60 \text { days }}=145 ; \mathrm{N}_{100 \text { days }}=135 ; \mathrm{N}_{180 \text { days }}=118$.

A

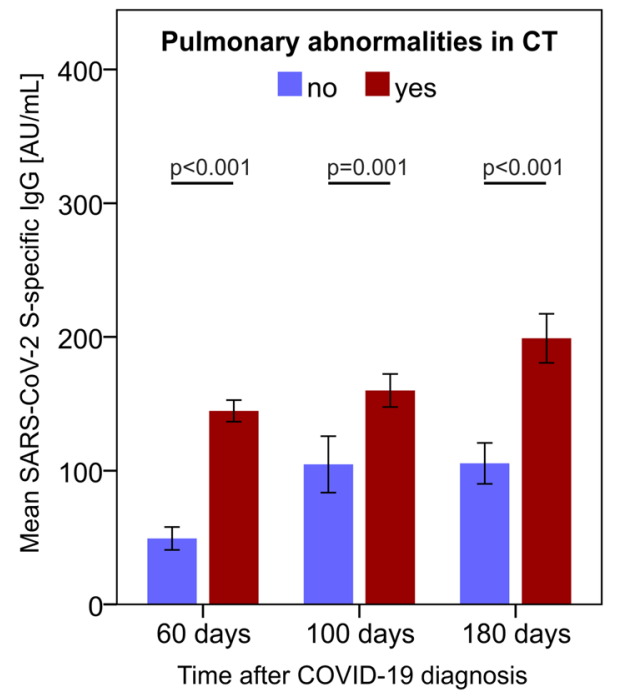

B

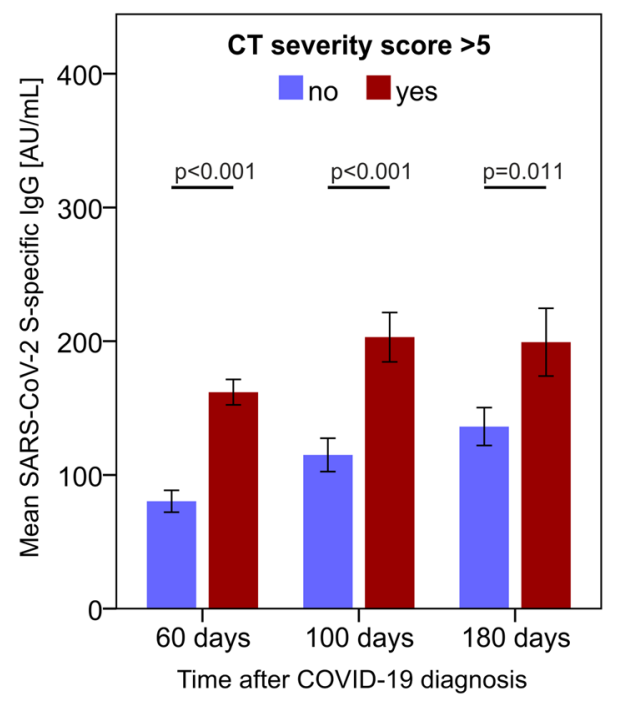

Figure 5. S-specific IgG levels correlate with pulmonary CT abnormalities. Structural pulmonary abnormalities were assessed with computed tomography (CT) and S-specific IgG serum concentrations according to (A) the presence of pulmonary CT abnormalities and (B) the presence of more pronounced CT abnormalities are shown. P-values were calculated with the Mann-Whitney $\mathrm{U}$ test. $\mathrm{N}_{60 \text { days }}=145\left(\mathrm{~N}_{\text {pulmonary findings }}=113\right.$, $\left.\mathrm{N}_{\text {CT severity score }>5}=72\right) ; \mathrm{N}_{100 \text { days }}=135\left(\mathrm{~N}_{\text {pulmonary findings }}=82, \mathrm{~N}_{\mathrm{CT} \text { severity score }>5}=40\right) ; \mathrm{N}_{180 \text { days }}=118\left(\mathrm{~N}_{\text {pulmonary findings }}=52\right.$, $\mathrm{N}_{\text {CT severity score }>5}=23$ ).

nary findings based on computed tomography (CT) assessments. We found that patients with pulmonary CT abnormalities at follow-up had higher levels of S-specific IgG concentrations in comparison to individuals who either lacked CT abnormalities or resolved such abnormalities by the follow-up (Fig. 5). We observed a similar correlative outcome when we stratified patients according to the severity of pulmonary CT abnormalities and compared S-specific IgG levels in individuals with no or very mild pulmonary CT abnormalities (severity score of $0-5$ points) to those with more prominent CT lung abnormalities (CT severity score $>5$ points). Importantly, pulmonary CT abnormalities could be linked to S-specific IgG levels at each of the 3 follow-up visits (Fig. 5). 


\begin{tabular}{|c|c|c|c|c|c|c|}
\hline \multirow[b]{3}{*}{ Parameter } & \multicolumn{2}{|c|}{ Univariate logistic regression } & \multicolumn{4}{|c|}{ Multivariate logistic regression } \\
\hline & \multirow{2}{*}{$\begin{array}{l}\text { CT abnormalities OR } \\
(95 \% \mathrm{CI})\end{array}$} & \multirow{2}{*}{$\begin{array}{l}\text { CT severity score }>5 \\
\text { OR }(95 \% \mathrm{CI})\end{array}$} & \multicolumn{2}{|c|}{ CT abnormalities } & \multicolumn{2}{|c|}{ CT severity score } \\
\hline & & & OR $(95 \% \mathrm{CI})$ & $\Delta$ deviance & OR $(95 \% \mathrm{CI})$ & $\Delta$ deviance \\
\hline Elevated CRP@V1 & $\begin{array}{l}5.7(1.9,21) \\
\mathrm{p}=0.0041\end{array}$ & $\begin{array}{l}6.1(2,19) \\
p=0.0046\end{array}$ & $\begin{array}{l}7.2(1.7,40) \\
\mathrm{p}=0.017\end{array}$ & $\begin{array}{l}-7.2 \\
p=0.01\end{array}$ & $\begin{array}{l}6.6(1.7,29) \\
p=0.019\end{array}$ & $\begin{array}{l}-7.5 \\
p=0.017\end{array}$ \\
\hline Sex male & $\begin{array}{l}3.9(1.8,8.8) \\
\mathrm{p}=0.0014\end{array}$ & $\begin{array}{l}3.7(1.3,12) \\
\mathrm{p}=0.017\end{array}$ & $\begin{array}{l}5(1.8,16) \\
p=0.0059\end{array}$ & $\begin{array}{l}-9.5 \\
p=0.0041\end{array}$ & $\begin{array}{l}4.9(1.4,23) \\
p=0.026\end{array}$ & $\begin{array}{l}-6.2 \\
\mathrm{p}=0.017\end{array}$ \\
\hline \# comorbidities & $\begin{array}{l}1.8(1.4,2.4) \\
\mathrm{p}=3.2 \mathrm{e}-05\end{array}$ & $\begin{array}{l}1.4(1.1,1.8) \\
\mathrm{p}=0.0046\end{array}$ & $\begin{array}{l}1.7(1.3,2.3) \\
p=0.00029\end{array}$ & $\begin{array}{l}-19 \\
\mathrm{p}=4.2 \mathrm{e}-05\end{array}$ & $\begin{array}{l}1.3(1,1.8) \\
p=0.026\end{array}$ & \begin{tabular}{|l|}
-5.3 \\
$\mathrm{p}=0.022$
\end{tabular} \\
\hline $\begin{array}{l}\text { SARS-CoV-2 anti-S-IgG } \\
\text { @V1 }\end{array}$ & $\begin{array}{l}1(1,1) \\
p=0.00049\end{array}$ & $\begin{array}{l}1(1,1) \\
p=0.0051\end{array}$ & $\begin{array}{l}1(1,1) \\
\mathrm{p}=0.021\end{array}$ & $\begin{array}{l}-5.7 \\
p=0.017\end{array}$ & $\begin{array}{l}1(1,1) \\
\mathrm{p}=0.026\end{array}$ & $\begin{array}{l}-6.2 \\
p=0.017\end{array}$ \\
\hline
\end{tabular}

Table 2. Results of univariate and multivariate risk modeling for parameters of the biosignature score. Correlations with presence of CT abnormalities at the 180 day follow-up were assessed by logistic regression. Significance of model terms was determined by Wald z-test. For the multivariate model, significance of particular terms was additionally assessed by likelihood-ratio tests (LRT, Chi ${ }^{2}$ test for $\Delta$ deviance $\neq 0$ ). p-values were corrected for multiple comparisons with the Benjamini-Hochberg method.

Assessment of S-specific IgG is useful to generate a pulmonary recovery score associated with lung recovery following COVID-19. Since S-specific IgG levels associated with impaired functional and structural pulmonary findings, we finally tested if S-specific serum IgG combined with other demographic and laboratory markers recorded at the 60-day follow-up could predict the risk of persistent lung pathologies in COVID-19 convalescents. We initially investigated the link between lung abnormality persistence by CT at the 180-day follow-up and known risk factors for COVID-19 severity, including male sex, the presence of comorbidities (as listed in Table 1), and serum levels of CRP and S-specific IgG determined at the 60-day follow-up. As shown in the Table 2, our univariate logistic model analysis uncovered a significant link between the investigated risk factors and structural lung recovery. Importantly, the tested parameters remained significant when combined in a multivariate fashion, suggesting an additive, non-colinear prediction value of non-recovering lung pathology for sex, comorbidity count, CRP levels, and S-specific IgG (Table 2). Thus, we combined various risk parameters to develop a pulmonary recovery score based on the linear coefficients of the multivariate logistic model (Fig. 6). Notably, the resulting lung recovery score demonstrated prediction accuracies exceeding $87 \%$ and a sensitivity over $84 \%$.

\section{Discussion}

SARS-CoV-2 causes a spectrum of manifestations that range from subclinical to severe and life-threatening infections. In patients with symptomatic disease, clinical complaints are diverse and include mild dry cough as well as loss of smell and taste, but also multiorgan dysfunction with hyperinflammation, acute respiratory distress syndrome, cardiovascular dysfunction, and coagulopathy ${ }^{38-40}$. A growing number of studies have shown that plasma biomarkers such as IL-6, ferritin, and D-dimers are useful for identifying patients at increased risk for such clinical complications, severe disease, and mortality ${ }^{38,41-44}$. However, we still lack parameters that can accurately predict delayed recovery in survivors of COVID-19 after hospital discharge ${ }^{45}$.

In the prospective COVID-19 follow-up study reported herein, we found that the magnitude of antibody response against the $\mathrm{S}$ glycoprotein of the causative infectious agent, SARS-CoV-2, is linked to pulmonary recovery based on laboratory evaluation, pulmonary function testing, and CT morphology assessments. Specifically, high concentrations of S-specific IgG, measured approximately 60 days after disease onset in outpatients followed up for COVID-19, identified individuals with abnormalities in pulmonary function and imaging studies persisting until 180 days after acute disease. Moreover, our data suggest that the levels of SARS-CoV-2 S-specific IgG, in conjunction with patient demographics and laboratory signs of inflammation, may be used to create a valuable recovery score for patient assessment in the early convalescent phase of the disease. This score allowed us to predict delayed pulmonary recovery with high accuracy, but it remains to be validated in independent patient cohorts.

In severe acute infections, a strong and dysregulated immune response characterized by a 'cytokine storm' can lead to multiorgan dysfunction ${ }^{46,47}$. This has been proposed for both the $1918 \mathrm{H} 1 \mathrm{~N} 1$ influenza pandemic ${ }^{48,49}$ and the ongoing SARS-CoV-2 pandemic ${ }^{50}$. However, other evidence suggests that cytokine production may rather be impaired during SARS-CoV-2 infection and that insufficient activation of innate and adaptive immunity may contribute to severe and fatal COVID-1951,52. Moreover, the inability to mount an appropriate IgG response against SARS-CoV-2 is linked to reduced survival. This association is best described for groups of patients who received the B cell-depleting monoclonal antibody, rituximab, within 6 months before contracting COVID-19. Specifically, patients with vasculitis, other rheumatic and inflammatory diseases or non-Hodgkin lymphoma were affected by higher morbidity and mortality from COVID-19 during the phase of hypogammaglobulinemia and B cell depletion ${ }^{53-57}$. Due to the design of our follow-up study, however, fatal cases of COVID-19 are not represented therein.

IL- 6 acts as a major inducer of innate immunity, enhances IgG production by B cells, and has recently emerged as an important biomarker for the early assessment of COVID-19 ${ }^{58}$. In our patient cohort, we found a robust relationship between SARS-CoV-2 S-specific IgG concentrations and persistent elevations of IL-6, ferritin, and hepcidin serum concentrations. Given the pleiotropic immunological functions of IL-6, we suggest that these 
A

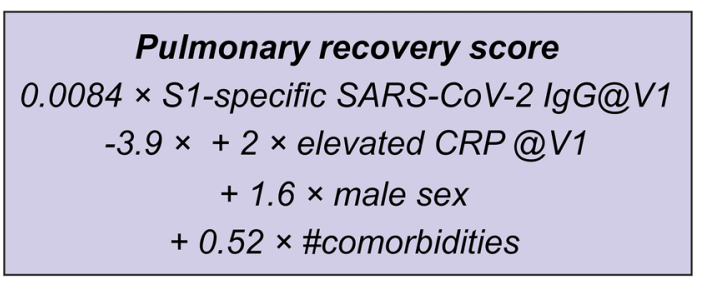

B

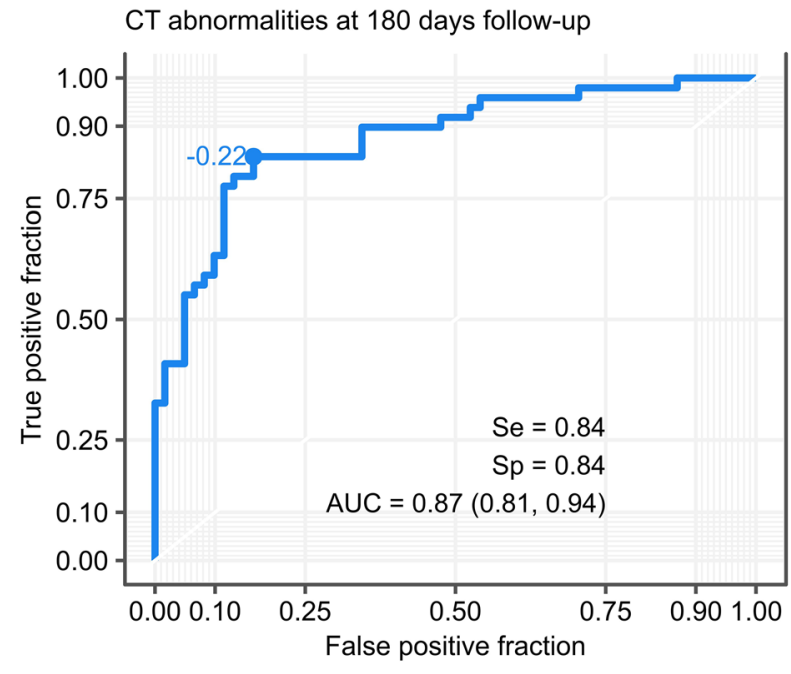

Total: $n=110$

Cases: $n=49$

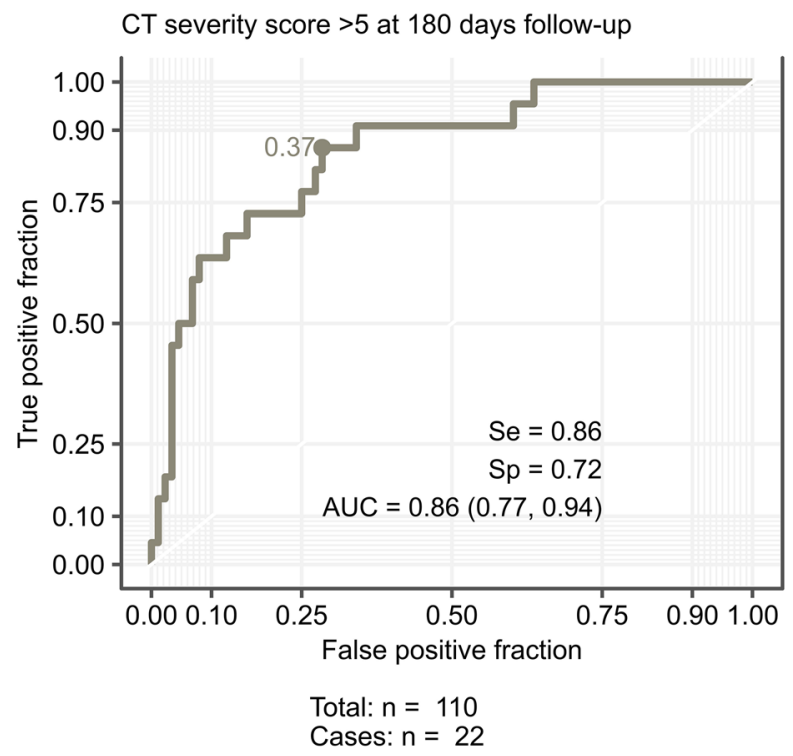

Figure 6. A pulmonary model incorporating early S-specific IgG measurement predicts pulmonary recovery at long-term follow-up. (A) We used S-specific IgG measurements acquired at the 60 days follow-up after RT-PCRbased diagnosis of COVID-19 to generate a score predicting structural lung recovery. (B) ROC analysis of the score used to predict the presence of pulmonary CT abnormalities and CT abnormalities with a CT severity score $>5$ at 180 days follow-up are shown.

relations are not attributable to statistical associations but rather to mechanistic links. Our data imply that COVID-19 is characterized by a broad and strong innate and adaptive immune response orchestrated by IL- 6 and other mediators. Furthermore, our results imply that the magnitude of immune response is interlinked with acute severity and impaired recovery. In this context, we herein demonstrate that persistent inflammation, as reflected by increased IL-6 at follow-up, and elevated serum concentrations of S-specific SARS-CoV-2 IgG are related to delayed pulmonary recovery, including impaired lung function and resolution of COVID-19-related pulmonary CT abnormalities.

Sustained production of IL-6 in severely ill COVID-19 patients may lead to prolonged activation of B cells and stimulate them to produce antibodies that increase both in terms of number and affinity ${ }^{27}$. Furthermore, viral antigens can be retained in secondary lymphoid tissues for several months, allowing affinity maturation to proceed even in the absence of reinfection or vaccination ${ }^{59}$. These antibodies against SARS-CoV-2 can have different specificities and, from an immunological standpoint, can be divided into neutralizing and non-neutralizing idiotypes ${ }^{30,60-63}$. Also, antibodies differ from each other in their immune function and rate of somatic hypermutation ${ }^{64-67}$. The majority of antibodies directed against SARS-CoV-2 are close to the germline configuration. Yet, extensive somatic hypermutation, an indication that antibodies have undergone affinity maturation in germinal centers, is linked to rapid recovery from COVID- $19^{59}$.

In the ongoing COVID-19 pandemic, data from many clinical studies have demonstrated the pleiotropic effects of immune responses against SARS-CoV-2 on patient clinical outcome. Our study adds to this knowledge and describes an independent patient cohort that has been followed up prospectively. Concretely, our data provide evidence that the link between immune activation and disease course is especially relevant for the lungs, which are a primary target for pathogen entry and replication. We used a broad clinical, laboratory, and imaging approach to follow up patients after COVID-19. Our data link the magnitude of the IgG response against the S glycoprotein of SARS-CoV-2 to COVID-19 severity and delayed disease recovery. Although the range of S-specific IgG levels overlapped between fully-recovered patients and those with persistent lung lesions during the observational study period, our results imply that implementing the measurement of SARS-CoV-2 specific IgG levels in the early covalescent phase of COVID-19 in clinical settings may help preselect for patients who might benefit from closer subsequent follow-up care. Thus, the combined use of laboratory and pulmonary function testing may help to avoid unnecessary CT in patients with otherwise unremarkable results. Therefore, limiting the use of CT to individual patients whose laboratory results and pulmonary functions remain altered, 
or to patients with a discrepancy between poor clinical recovery and unremarkable laboratory and lung function testing, may be a feasible approach that is effective from the perspective of costs, resources, and X-ray exposure. Our study thus provides a framework for systematic and resource sparing follow-up care of larger numbers of COVID-19 patients and deserves validation in independent patient cohorts.

The CovILD study was specifically designed to follow up adult patients after symptomatic COVID-19 and, as such, has several limitations. First, at both ends of the spectrum, asymptomatic and fatal infections with SARS$\mathrm{CoV}-2$ are not represented in our patient cohort. Rather, approximately three-quarters of the patients in our study were hospitalized for COVID-19, and approximately two-thirds of hospitalized patients required oxygen supplementation without or with subsequent invasive ventilation. Nevertheless, patients with mild to critical disease are equally represented in our cohort. Furthermore, patients with moderate, severe, and critical disease courses are the ones that qualify the most to be screened for potential long-term impairment after COVID-19. Second, patients $<18$ years of age met an exclusion criterion and are thus not represented in our study. However, COVID-19 is typically a mild disease in children who therefore may not require extensive work-up by laboratory, pulmonary function, and imaging studies ${ }^{68}$. Third, although our study is multicentric by design, our cohort is rather small. Yet, due to the extensive characterization of the CovILD cohort, the sample size was large enough to identify new robust associations between levels of S-specific IgG and clinical, functional, and CT morphologic findings, resulting in the implementation of a novel pulmonary recovery score.

\section{Methods \\ Patients and study design. The development of interstitial lung disease (ILD) in patients with severe SARS-CoV-2 infection (CovILD) study ${ }^{45}$ is an ongoing prospective, multicenter, observational cohort trial aim- ing to systematically follow symptomatic patients after COVID-19 (ClinicalTrials.gov number, NCT04416100). The initial diagnosis of COVID-19 was based on typical clinical symptoms and a positive RT-PCR for SARS- CoV-2 obtained from a nasopharyngeal or oropharyngeal swab using the Altona RealStar ${ }^{\circledR}$ SARS-CoV-2 PCR RT-PCR kit 1.0, the Cepheid Xpert ${ }^{\oplus}$ Xpress SARS-CoV-2 test, or the Roche cobas ${ }^{\circledast}$ SARS-CoV-2 test. As a con- firmatory test, the Altona or the Roche SARS-CoV-2 RT-PCR was used as needed to definitively establish the diagnosis and guide the ending of isolation. The CovILD study was approved by the local ethics committee at the Medical University Innsbruck (EK Nr: 1103/2020). All research was performed in following relevant guidelines and regulations and is in accordance with the Declaration of Helsinki. Written informed consent was obtained from all study participants and a total of 145 COVID-19 convalescents who previously suffered from mild to critical disease were included in the analysis presented herein as detailed in the Online Supplement.}

Measurement of S-specific IgG levels. IgG antibodies against the S protein of SARS-CoV-2 were quantified with LIAISON ${ }^{\oplus}$ SARS-CoV-2 S1/S2 IgG CLIA (DiaSorin, Italy) and expressed as (AU/ml) using a cut-off of $16.85 \mathrm{AU} / \mathrm{ml}$ validated as detailed in the Online Supplement.

Categorization of clinical severity. We divided the study participants into 4 groups according to clinical disease severity during acute COVID-19 as follows: (1) mild disease: outpatient management without the need for hospitalization; (2) moderate disease: hospitalization without the need for respiratory support; (3) severe disease: hospitalization with the need for oxygen supply; (4) critical disease: ICU treatment with respiratory failure and the need for mechanical ventilation.

Blood sampling and further analysis. Blood samples were taken via routine peripheral vein puncture and analyzed by standardized ISO-certified procedures. Native or heparinized blood was separated via centrifugation at $300 \times g$ to collect serum or plasma, respectively, as previously described in detail ${ }^{69}$. Serum samples were frozen at $-30{ }^{\circ} \mathrm{C}$ after centrifugation and stored until batch-wise analysis was performed as described in the Online Supplement.

Analysis of lung involvement with computed tomography. 60 days after the diagnosis of COVID19 , we evaluated all study participants with a low-dose (100 kVp tube potential) computed tomography (CT) scan of the chest without the use of an iodine contrast agent as described ${ }^{44}$. CT was acquired on a 128 slice multidetector CT hardware with a $38.4 \times 0.6 \mathrm{~mm}$ collimation and spiral pitch factor of 1.1 (SOMATOM Definition Flash, Siemens Healthineers, Erlangen, Germany). CT images were evaluated as detailed in the Online Supplement.

Statistical analysis. Statistical analyses were performed with statistical analysis software package (IBM SPSS Statistics version 26.0, IBM, USA) and R programming suite version 4.0.3. Descriptive statistics included tests for homoscedasticity and data distribution (Levene test, Kolmogorov-Smirnov test, Shapiro-Wilk test, and density blot/histogram analysis). According to explorative data analysis, we used the following tests: MannWhitney $U$ test, Kruskal-Wallis and Friedman's test for group comparisons of continuous data, Fisher's exact test, or Chi-Square test for binary and categorical data. Multiple testing was adjusted by the Sidak formula as appropriate. For the development of a pulmonary recovery score, we used logistic regression as detailed in the Online Supplement.

\section{Data availability}

The datasets generated during and/or analysed during the current study are available from the corresponding authors upon reasonable request. 
Received: 13 July 2021; Accepted: 16 February 2022

Published online: 07 March 2022

\section{References}

1. Wiersinga, W. J., Rhodes, A., Cheng, A. C., Peacock, S. J. \& Prescott, H. C. Pathophysiology, transmission, diagnosis, and treatment of coronavirus disease 2019 (COVID-19): A review. JAMA 324, 782-793. https://doi.org/10.1001/jama.2020.12839 (2020).

2. Streeck, H. et al. Infection fatality rate of SARS-CoV2 in a super-spreading event in Germany. Nat. Commun. 11, 5829. https:// doi.org/10.1038/s41467-020-19509-y (2020).

3. Buitrago-Garcia, D. et al. Occurrence and transmission potential of asymptomatic and presymptomatic SARS-CoV-2 infections: A living systematic review and meta-analysis. PLoS Med. 17, e1003346. https://doi.org/10.1371/journal.pmed.1003346 (2020).

4. Tay, M. Z., Poh, C. M., Renia, L., MacAry, P. A. \& Ng, L. F. P. The trinity of COVID-19: Immunity, inflammation and intervention. Nat. Rev. Immunol. 20, 363-374. https://doi.org/10.1038/s41577-020-0311-8 (2020).

5. Nishiga, M., Wang, D. W., Han, Y., Lewis, D. B. \& Wu, J. C. COVID-19 and cardiovascular disease: From basic mechanisms to clinical perspectives. Nat. Rev. Cardiol. 17, 543-558. https://doi.org/10.1038/s41569-020-0413-9 (2020).

6. Duggal, N. K. \& Emerman, M. Evolutionary conflicts between viruses and restriction factors shape immunity. Nat. Rev. Immunol. 12, 687-695. https://doi.org/10.1038/nri3295 (2012).

7. Merad, M. \& Martin, J. C. Pathological inflammation in patients with COVID-19: A key role for monocytes and macrophages. Nat. Rev. Immunol. 20, 355-362. https://doi.org/10.1038/s41577-020-0331-4 (2020).

8. Cao, X. COVID-19: Immunopathology and its implications for therapy. Nat. Rev. Immunol. 20, 269-270. https://doi.org/10.1038/ s41577-020-0308-3 (2020).

9. Sariol, A. \& Perlman, S. Lessons for COVID-19 immunity from other coronavirus infections. Immunity 53, 248-263. https://doi. org/10.1016/j.immuni.2020.07.005 (2020).

10. Zhao, J. et al. Antibody responses to SARS-CoV-2 in patients with novel coronavirus disease 2019. Clin. Infect. Dis. 71, 2027-2034. https://doi.org/10.1093/cid/ciaa344 (2020).

11. Braun, J. et al. SARS-CoV-2-reactive T cells in healthy donors and patients with COVID-19. Nature 587, 270-274. https://doi.org/ 10.1038/s41586-020-2598-9 (2020).

12. Meckiff, B. J. et al. Imbalance of regulatory and cytotoxic SARS-CoV-2-reactive CD4(+) T cells in COVID-19. Cell 183, 1340-1353. https://doi.org/10.1016/j.cell.2020.10.001 (2020).

13. Vashist, S. K. In vitro diagnostic assays for COVID-19: Recent advances and emerging trends. Diagnostics https://doi.org/10.3390/ diagnostics10040202 (2020).

14. Espejo, A. P. et al. Review of current advances in serologic testing for COVID-19. Am. J. Clin. Pathol. 154, 293-304. https://doi. org/10.1093/ajcp/aqaa112 (2020).

15. Irsara, C. et al. Clinical validation of the Siemens quantitative SARS-CoV-2 spike IgG assay (sCOVG) reveals improved sensitivity and a good correlation with virus neutralization titers. Clin. Chem. Lab. Med. https://doi.org/10.1515/cclm-2021-0214 (2021).

16. Irsara, C. et al. Evaluation of four commercial, fully automated SARS-CoV-2 antibody tests suggests a revision of the Siemens SARS-CoV-2 IgG assay. Clin. Chem. Lab. Med. https://doi.org/10.1515/cclm-2020-1758 (2020).

17. Batra, M. et al. Role of IgG against N-protein of SARS-CoV2 in COVID19 clinical outcomes. Sci. Rep. 11, 3455. https://doi.org/ 10.1038/s41598-021-83108-0 (2021).

18. Ma, H. et al. Serum IgA, IgM, and IgG responses in COVID-19. Cell. Mol. Immunol. 17, 773-775. https://doi.org/10.1038/s41423020-0474-z (2020).

19. Klingler, J. et al. Role of IgM and IgA antibodies in the neutralization of SARS-CoV-2. J. Infect. Dis. https://doi.org/10.1093/infdis/ jiaa784 (2020).

20. Yu, H. Q. et al. Distinct features of SARS-CoV-2-specific IgA response in COVID-19 patients. Eur. Respir. J. https://doi.org/10. 1183/13993003.01526-2020 (2020).

21. Iyer, A. S. et al. Persistence and decay of human antibody responses to the receptor binding domain of SARS-CoV-2 spike protein in COVID-19 patients. Sci. Immunol. https://doi.org/10.1126/sciimmunol.abe0367 (2020).

22. Sterlin, D. et al. IgA dominates the early neutralizing antibody response to SARS-CoV-2. Sci. Transl. Med. https://doi.org/10.1126/ scitranslmed.abd2223 (2020).

23. Wang, Z. et al. Enhanced SARS-CoV-2 neutralization by dimeric IgA. Sci. Transl. Med. https://doi.org/10.1126/scitranslmed.abf15 55 (2020).

24. Orth-Holler, D., Eigentler, A., Stiasny, K., Weseslindtner, L. \& Most, J. Kinetics of SARS-CoV-2 specific antibodies (IgM, IgA, $\mathrm{IgG}$ ) in non-hospitalized patients four months following infection. J. Infect. 82, 282-327. https://doi.org/10.1016/j.jinf.2020.09. 015 (2020).

25. Isho, B. et al. Persistence of serum and saliva antibody responses to SARS-CoV-2 spike antigens in COVID-19 patients. Sci. Immunol. https://doi.org/10.1126/sciimmunol.abe5511 (2020).

26. Ripperger, T. J. et al. Orthogonal SARS-CoV-2 serological assays enable surveillance of low-prevalence communities and reveal durable humoral immunity. Immunity 53, 925-933. https://doi.org/10.1016/j.immuni.2020.10.004 (2020).

27. Pichler, D. et al. Marked increase in avidity of severe acute respiratory syndrome coronavirus-2 (SARS-CoV-2) antibodies $7-8$ months after infection is not diminished in old age. J. Infect. Dis. https://doi.org/10.1093/infdis/jiab300 (2021).

28. Yao, L. et al. Persistence of antibody and cellular immune responses in COVID-19 patients over nine months after infection. J. Infect. Dis. https://doi.org/10.1093/infdis/jiab255 (2020).

29. Figueiredo-Campos, P. et al. Seroprevalence of anti-SARS-CoV-2 antibodies in COVID-19 patients and healthy volunteers up to 6 months post disease onset. Eur. J. Immunol. 50, 2025-2040. https://doi.org/10.1002/eji.202048970 (2020).

30. Gaebler, C. et al. Evolution of antibody immunity to SARS-CoV-2. Nature https://doi.org/10.1038/s41586-021-03207-w (2020).

31. Zohar, T. et al. Compromised humoral functional evolution tracks with SARS-CoV-2 mortality. Cell 183, 1508-1519. https://doi. org/10.1016/j.cell.2020.10.052 (2020).

32. De Giorgi, V. et al. Naturally acquired SARS-CoV-2 immunity persists for up to 11 months following infection. J. Infect. Dis. https:// doi.org/10.1093/infdis/jiab295 (2020).

33. Lynch, K. L. et al. Magnitude and kinetics of anti-severe acute respiratory syndrome coronavirus 2 antibody responses and their relationship to disease severity. Clin. Infect. Dis. 72, 301-308. https://doi.org/10.1093/cid/ciaa979 (2020).

34. Haddad, N. S. et al. Elevated SARS-CoV-2 antibodies distinguish severe disease in early COVID-19 infection. BioRxiv https://doi. org/10.1101/2020.12.04.410589 (2020).

35. Phipps, W. S. et al. SARS-CoV-2 antibody responses do not predict covid-19 disease severity. Am. J. Clin. Pathol. 154, 459-465. https://doi.org/10.1093/ajcp/aqaa123 (2020).

36. Ng, K. W. et al. Preexisting and de novo humoral immunity to SARS-CoV-2 in humans. Science 370, 1339-1343. https://doi.org/ 10.1126/science.abe1107 (2020).

37. Weisberg, S. P. et al. Distinct antibody responses to SARS-CoV-2 in children and adults across the COVID-19 clinical spectrum. Nat. Immunol. 22, 25-31. https://doi.org/10.1038/s41590-020-00826-9 (2020).

38. Al-Samkari, H. et al. COVID-19 and coagulation: Bleeding and thrombotic manifestations of SARS-CoV-2 infection. Blood 136, 489-500. https://doi.org/10.1182/blood.2020006520 (2020). 
39. Li, H. et al. SARS-CoV-2 and viral sepsis: Observations and hypotheses. Lancet 395, 1517-1520. https://doi.org/10.1016/S01406736(20)30920-X (2020).

40. Zhang, Y. et al. Coagulopathy and antiphospholipid antibodies in patients with covid-19. N. Engl. J. Med. 382, e38. https://doi.org/ 10.1056/NEJMc2007575 (2020).

41. Abers, M. S. et al. An immune-based biomarker signature is associated with mortality in COVID-19 patients. JCI Insight https:// doi.org/10.1172/jci.insight.144455 (2020).

42. Broman, N. et al. IL-6 and other biomarkers as predictors of severity in COVID-19. Ann. Med. 53, 410-412. https://doi.org/10. $1080 / 07853890.2020 .1840621(2020)$.

43. Liu, T. et al. The role of interleukin-6 in monitoring severe case of coronavirus disease 2019. EMBO Mol. Med. 12, e12421. https:// doi.org/10.15252/emmm.202012421 (2020).

44. Sonnweber, T. et al. Persisting alterations of iron homeostasis in COVID-19 are associated with non-resolving lung pathologies and poor patients' performance: a prospective observational cohort study. Respir. Res. 21, 276. https://doi.org/10.1186/s12931020-01546-2 (2020).

45. Sonnweber, T. et al. Cardiopulmonary recovery after COVID-19 - an observational prospective multi-center trial. Eur. Respir. J. https://doi.org/10.1183/13993003.03481-2020 (2020).

46. Fajgenbaum, D. C. \& June, C. H. Cytokine storm. N. Engl. J. Med. 383, 2255-2273. https://doi.org/10.1056/NEJMra2026131 (2020).

47. Mangalmurti, N. \& Hunter, C. A. Cytokine storms: Understanding COVID-19. Immunity 53, 19-25. https://doi.org/10.1016/j. immuni.2020.06.017 (2020).

48. Kash, J. C. et al. Genomic analysis of increased host immune and cell death responses induced by 1918 influenza virus. Nature 443, 578-581. https://doi.org/10.1038/nature05181 (2006).

49. Kobasa, D. et al. Aberrant innate immune response in lethal infection of macaques with the 1918 influenza virus. Nature 445, 319-323. https://doi.org/10.1038/nature05495 (2007).

50. Moore, J. B. \& June, C. H. Cytokine release syndrome in severe COVID-19. Science 368, 473-474. https://doi.org/10.1126/scien ce.abb8925 (2020).

51. Leisman, D. E. et al. Cytokine elevation in severe and critical COVID-19: A rapid systematic review, meta-analysis, and comparison with other inflammatory syndromes. Lancet Respir. Med. 8, 1233-1244. https://doi.org/10.1016/S2213-2600(20)30404-5 (2020).

52. Remy, K. E. et al. Severe immunosuppression and not a cytokine storm characterizes COVID-19 infections. JCI Insight https:// doi.org/10.1172/jci.insight.140329 (2020).

53. Tepasse, P. R. et al. Persisting SARS-CoV-2 viraemia after rituximab therapy: Two cases with fatal outcome and a review of the literature. Br. J. Haematol. 190, 185-188. https://doi.org/10.1111/bjh.16896 (2020).

54. Loarce-Martos, J. et al. High rates of severe disease and death due to SARS-CoV-2 infection in rheumatic disease patients treated with rituximab: A descriptive study. Rheumatol. Int. 40, 2015-2021. https://doi.org/10.1007/s00296-020-04699-x (2020).

55. Duléry, R. et al. Prolonged in-hospital stay and higher mortality after Covid-19 among patients with non-Hodgkin lymphoma treated with B-cell depleting immunotherapy. Am. J. Hematol. 96, 934-944. https://doi.org/10.1002/ajh.26209 (2021).

56. Kronbichler, A. et al. The COVID-19 pandemic and ANCA-associated vasculitis: Reports from the EUVAS meeting and EUVAS education forum. Autoimmun. Rev. https://doi.org/10.1016/j.autrev.2021.102986 (2021).

57. Calderón-Parra, J. et al. Incidence, clinical presentation, relapses and outcome of SARS-CoV-2 infection in patients treated with anti-CD20 monoclonal antibodies. Clin. Infect. Dis. https://doi.org/10.1093/cid/ciab700 (2021).

58. Hirano, T. et al. Complementary DNA for a novel human interleukin (BSF-2) that induces B lymphocytes to produce immunoglobulin. Nature 324, 73-76. https://doi.org/10.1038/324073a0 (1986).

59. Chen, Y. et al. Quick COVID-19 healers sustain anti-SARS-CoV-2 antibody production. Cell 183, 1496-1507. https://doi.org/10. 1016/j.cell.2020.10.051 (2020).

60. Barnes, C. O. et al. Structures of human antibodies bound to SARS-CoV-2 spike reveal common epitopes and recurrent features of antibodies. Cell 182, 828-842. https://doi.org/10.1016/j.cell.2020.06.025 (2020).

61. Brouwer, P. J. M. et al. Potent neutralizing antibodies from COVID-19 patients define multiple targets of vulnerability. Science 369, 643-650. https://doi.org/10.1126/science.abc5902 (2020).

62. Prevost, J. et al. Cross-sectional evaluation of humoral responses against SARS-CoV-2 spike. Cell. Rep. Med. 1, 100126. https:// doi.org/10.1016/j.xcrm.2020.100126 (2020).

63. Nielsen, S. C. A. et al. Human B cell clonal expansion and convergent antibody responses to SARS-CoV-2. Cell Host Microbe 28, 516-525. https://doi.org/10.1016/j.chom.2020.09.002 (2020).

64. Atyeo, C. et al. Distinct early serological signatures track with SARS-CoV-2 survival. Immunity 53, 524-532. https://doi.org/10. 1016/j.immuni.2020.07.020 (2020).

65. Bartsch, Y. C. et al. Discrete SARS-CoV-2 antibody titers track with functional humoral stability. Nat. Commun. 12, 1018. https:// doi.org/10.1038/s41467-021-21336-8 (2020).

66. Kreer, C. et al. Longitudinal isolation of potent near-germline SARS-CoV-2-neutralizing antibodies from COVID-19 patients. Cell 182, 1663-1673. https://doi.org/10.1016/j.cell.2020.08.046 (2020).

67. Robbiani, D. F. et al. Convergent antibody responses to SARS-CoV-2 in convalescent individuals. Nature 584, 437-442. https:// doi.org/10.1038/s41586-020-2456-9 (2020).

68. Gotzinger, F. et al. COVID-19 in children and adolescents in Europe: A multinational, multicentre cohort study. Lancet Child. Adolesc. Health 4, 653-661. https://doi.org/10.1016/S2352-4642(20)30177-2 (2020).

69. Sonnweber, T. et al. Impact of iron treatment on immune effector function and cellular iron status of circulating monocytes in dialysis patients. Nephrol. Dial Transplant. 26, 977-987. https://doi.org/10.1093/ndt/gfq483 (2011).

\section{Acknowledgements}

This project was funded by the Austrian Science Fund (FWF), project P33062 (to M.N.). J. LR. acknowledges receiving financial support from the 'Land Tirol Forschungsförderung' (Project GZ 71934). I. Tancevski acknowledges having been awarded an Investigator-Initiated Study (IIS) grant by 'Boehringer Ingelheim' (IIS 1199-0424). We gratefully acknowledge the commitment of the staff, providers, and personnel at the Central Institute of Clinical and Chemical Laboratory Diagnostics at the University Hospital of Innsbruck, the Departments of Internal Medicine and Radiology at the Medical University of Innsbruck, and of the Institutions of the Hospital of Zams who contributed to this study.

\section{Author contributions}

T.S. and M.N. had the idea for and designed the study presented herein. T.S., J.L.R. and I.Tancevski were responsible for the conception and design of the CovILD study. T.S. and P.T. performed the statistical analysis. T.S., S.S., A.P., A.B., R.B.W., S.K., A.S., D.H., V.P., J.L.R. and I.Tancevski recruited and evaluated patients. A.L., C.S., and G.Widmann analyzed CT images. G.Weiss interpreted data and provided essential intellectual input. A.M.M., L.v.R., P.G., and I.Theurl contributed to correcting and improving the manuscript. M.Z., S.B., K.T., H.N., W.K., 
C.G. and M.N. collected and analyzed blood samples. M.N. and T.S. wrote the manuscript. J.E.M. reviewed and edited the manuscript. All authors approved the final manuscript.

\section{Competing interests}

The authors declare no competing interests.

\section{Additional information}

Supplementary Information The online version contains supplementary material available at https://doi.org/ 10.1038/s41598-022-07489-6.

Correspondence and requests for materials should be addressed to M.N. or T.S.

Reprints and permissions information is available at www.nature.com/reprints.

Publisher's note Springer Nature remains neutral with regard to jurisdictional claims in published maps and institutional affiliations.

(c) (i) Open Access This article is licensed under a Creative Commons Attribution 4.0 International License, which permits use, sharing, adaptation, distribution and reproduction in any medium or format, as long as you give appropriate credit to the original author(s) and the source, provide a link to the Creative Commons licence, and indicate if changes were made. The images or other third party material in this article are included in the article's Creative Commons licence, unless indicated otherwise in a credit line to the material. If material is not included in the article's Creative Commons licence and your intended use is not permitted by statutory regulation or exceeds the permitted use, you will need to obtain permission directly from the copyright holder. To view a copy of this licence, visit http://creativecommons.org/licenses/by/4.0/.

(C) The Author(s) 2022 